\title{
Current Status of Exposed Endoscopic Full-Thickness Resection and Further Development of Non-Exposed Endoscopic Full-Thickness Resection
}

\author{
Hirohito Mori ${ }^{a, c}$ Asadur Rahman ${ }^{b}$ Hideki Kobara $^{a}$ Shintaro Fujihara ${ }^{a}$ \\ Noriko Nishiyama $^{a}$ Maki Ayaki $^{a}$ Tae Matsunaga ${ }^{a}$ Masanori Murakami ${ }^{c}$ \\ Tsumomu Masaki ${ }^{a}$ \\ a Department of Gastroenterology and Neurology, and bepartment of Pharmacology, Faculty of Medicine, Kagawa \\ University, Takamatsu, and 'Department of Gastroenterological Surgery, Ehime Rosai Hospital, Niihama, Japan
}

\section{Key Words}

Endoscopic full-thickness resection · Submucosal tunnel endoscopic resection $\cdot$ Non-exposed $\cdot$ Suturing devices

\begin{abstract}
Endoscopic full-thickness resection (EFTR) is a procedure that makes it possible to access the lesions that are on the wall of the digestive tract via the shortest distance through the mouth. Because of the ultra-minimal invasive nature of the treatment, pure EFTR is a highly promising surgical procedure that allows the radical excision of full-thickness layers of digestive tract tumors using only a flexible endoscope. There are 2 types of EFTR methods: exposed and non-exposed. Considering the risks of contracting infection and intraperitoneal dissemination of tumor cells, non-exposed EFTR is an ideal method. However, a number of issues remain unresolved, including the method for performing a full-thickness suture under endoscopic view and the challenge of securing the operating field in the case of gastrointestinal tract collapse. Moreover, advances in the development of equipment such as full-thickness suturing devices would be helpful to make this therapeutic procedure the most minimally invasive endoscopic surgery ever.
\end{abstract}

(c) 2016 S. Karger AG, Basel (c) 2016 S. Karger AG, Basel

0012-2823/16/0951-0006\$39.50/0

\section{Introduction}

To date, flexible endoscopic examination has been used mainly for diagnosis by using gastrointestinal fiberscopes and colonic fiberscopes, and for the biopsy of tissue samples from lesion sites. Since the development of electronic endoscopes, polypectomy using snare resection, endoscopic mucosal resections, and strip biopsy using local injections have been reported. Currently, these methods have been modified and developed into endoscopic submucosal dissection (ESD), which is an extremely minimally invasive treatment for gastrointestinal malignancies, and which utilizes electric scalpels for exclusive use with flexible endoscopes [1-4]. Esophageal, gastric, and colorectal ESDs are now covered by health insurance and have become established therapeutic procedures for treating early esophageal cancers, gastric cancers as well as colorectal cancers.

Kalloo et al. [5] performed a transgastric observation of the peritoneal cavity by making an incision in the gastric wall in 2004; since then various surgical procedures using flexible endoscopes have been reported. Among these, the natural orifice translumenal endoscopic surgery (NOTES) using a flexible endoscope is an ultra-minimally invasive surgical method that makes no surgical

\section{KARGER}

E-Mail karger@karger.com

www.karger.com/dig
Hirohito Mori, MD, PhD

Department of Gastroenterology and Neurology

Faculty of Medicine, Kagawa University

1750-1 Ikenobe, Miki-cho, Kita-gun, Kagawa 761-0793 (Japan)

E-Mail hiro4884@med.kagawa-u.ac.jp 
wound or scar after operation. However, due to the several limitations that have been reported in many ex vivo and in vivo experiments relating to NOTES, it was difficult for almost all of surgeons, endoscopists and gastroenterologists to agree and accept this immature surgical procedure. Hence, reports with regard to NOTES have drastically decreased. But, among such immature surgical procedures, only endoscopic full-thickness resection (EFTR) has been reported to be highly feasible and acceptable procedures. EFTR is a full-thickness local excision of the gastrointestinal tract, which is carried out using devices and techniques aimed at performing ESD. EFTR is performed using only flexible endoscopes for the treatment of gastrointestinal epithelial malignancies such as gastric cancers, colorectal cancers or submucosal tumors(SMTs) likegastrointestinal stromal tumors(GISTs). However, a number of issues are yet unsolved, including the method for performing full-thickness suturing at the resection site, as well as preventing the complication of digestive tract collapse [6].

There are 2 types of EFTR: exposed EFTR and nonexposed EFTR (Neo-EFTR). Considering the risks of infection and intraperitoneal dissemination of the tumor cells, neo-EFTR is recommended as the best method [7].

\section{Materials and Methods}

\section{Search Strategy and Overview}

Relevant prospective or retrospective studies were identified by searching the Medline, PubMed. The authors inspected all identified references individually.

Search queries were as follows: 'endoscopic full-thickness resection or EFTR' and 'submucosal tunnel endoscopic resection or STER' and 'over-the-scope clip or OTSC', and 'suturing device or closure' and 'laparoscopy and endoscopy cooperative surgery or LECS'.

\section{Selection Criteria of the Study}

Studies were selected if the primary outcome and secondary outcomes were clearly mentioned. Case reports/ series and animal studies were included.

\section{Indication for Full-Thickness Resection}

If lymphadenectomy is not required for GISTs, it might be a good indication for full-thickness resection for the following reasons: EFTR can be safely applied for clinical treatment; the resection line can be identified from inside the gastric cavity; infection can be controlled by preventing intraperitoneal exposure of the gastric juice; there is no risk of dissemination of the tumor cells; the tumor's size allows the resected lesion to be retrieved through the mouth [6]. For pure exposed EFTR, GIST is a good candidate that allows the retrieval of the resected lesions approximately $3 \mathrm{~cm}$ in diameter through the mouth [8].

For Neo-EFTR, the indications for the procedure are as follows: patients who are not subjected to ESD because of epithelial tumors with virtually no lymph node metastasis but with ulcer scar due to a depth of invasion classified as M or SM1; patients who are not subjected to ESD because of an early-stage gastric cancer developing on a benign ulcer; patients with early-stage gastric cancers at complicated locations such as in the gastric fundus, which are likely to be perforated and cause peritoneal dissemination of cancer cells.

\section{Current Techniques of Full-Thickness Resection}

\section{Exposed EFTR (fig. 1)}

Wang et al. [9] reported a retrospective comparison of patients $(\mathrm{n}=35)$ who underwent exposed EFTR with patients $(\mathrm{n}=33)$ who underwent laparoscopic-assisted surgery (LAC; a total of 68 patients with GISTs smaller than $2 \mathrm{~cm}$ in diameter that originated from the muscularis propria, MP). They concluded that complications were lower in the EFTR group than in the LAC group, which had 3 wound infections and 1 leakage. Moreover, EFTR was safer than LAC for GISTs under $2 \mathrm{~cm}$. However, this method is designed for tumors with a resection diameter as small as $13 \pm 5 \mathrm{~mm}$ and with a size that allows for closure with a hemoclip.

In a study conducted on 62 cases of SMTs of the gastric fundus, Lu et al. [10] carried out a retrospective comparison of the method of EFTR, thread-with-clip method, and loop-assisted method. They reported that operation time was significantly shorter in EFTR with both the thread-with-clip method and loop-assisted method. There were no fatal cases, and the surgical procedure was reportedly safe. However, 20-30\% of the excised tissue consisted of leiomyoma. The tumor was an SMT with a diameter as small as approximately $20 \mathrm{~mm}$. High fever was also observed following EFTR, suggesting the onset of peritonitis. The reason for the high resection rate (97\%) was that these EFTR procedures were performed by highly experienced endoscopists at the high-volume center. Hence, it cannot be affirmed that EFTR is easy to perform 

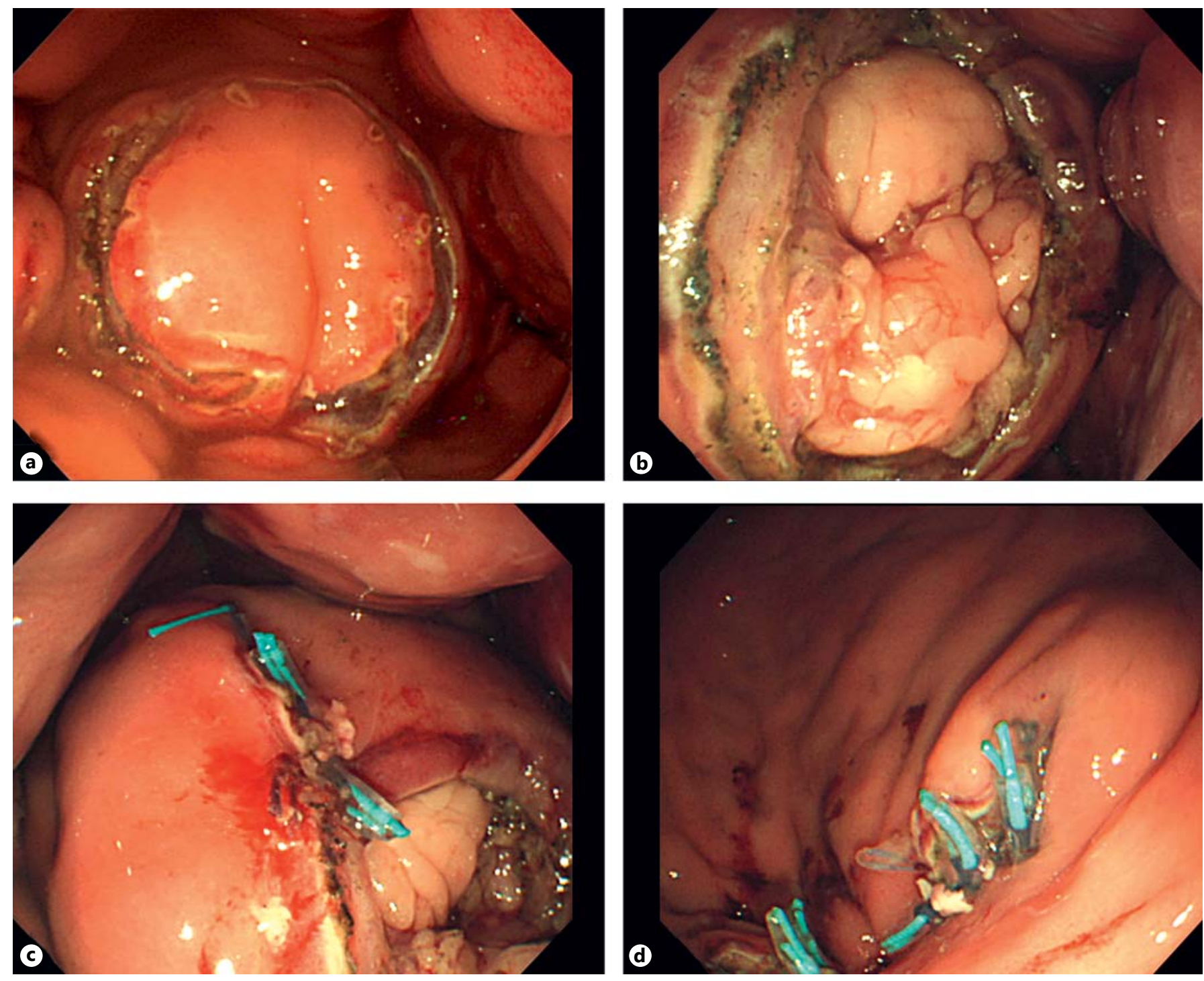

Fig. 1. Pure exposed EFTR of a $40 \mathrm{~mm}$ gastric pseudo tumor. ESD procedure was used to deepen the incision until it reached just above the muscle layer (a). Cutting the remaining layers (muscular

in terms of technical methodologies. Although it is tough to make any conclusion regarding the long-term outcomes, the small size of GISTs may lead to reduced peritoneal dissemination, whereas exposed EFTR may lead to infection and peritoneal dissemination. In addition, there is no information about the deterioration of the conditions in the operating field as a result of the destruction of the gastrointestinal tract associated with a large SMT [11]. The guidelines published by the Japan Society of Clinical Oncology stated that in the case of a GISTs over $20 \mathrm{~mm}$ in diameter, which is a relative indication for tumor resection, a clinical introduction of exposed EFTR is layer and serosal), exposed EFTR was completed (b). Full-thickness suturing using a DBSS (c). Ten-stitch sutures were performed at $3 \mathrm{~mm}$ intervals (d).

associated with safety problems, infection, and dissemination. As a result, its utility remains unclear. In addition, closure of the resection opening using only a hemoclip may be impossible, if the tumor is large in size.

Cai et al. [6] previously reported that the resection opening of EFTR could be closed with a hemoclip; however, some factors should be taken under considerations, for example, the issue of training and the need for the development of suturing instruments and devices $[12,13]$.

Guo et al. [13] reported 23 cases of exposed EFTR with a defective closure identified using an OTSC system (Ovesco Endoscopy GmbH, Tuebingen, Germany) retro- 

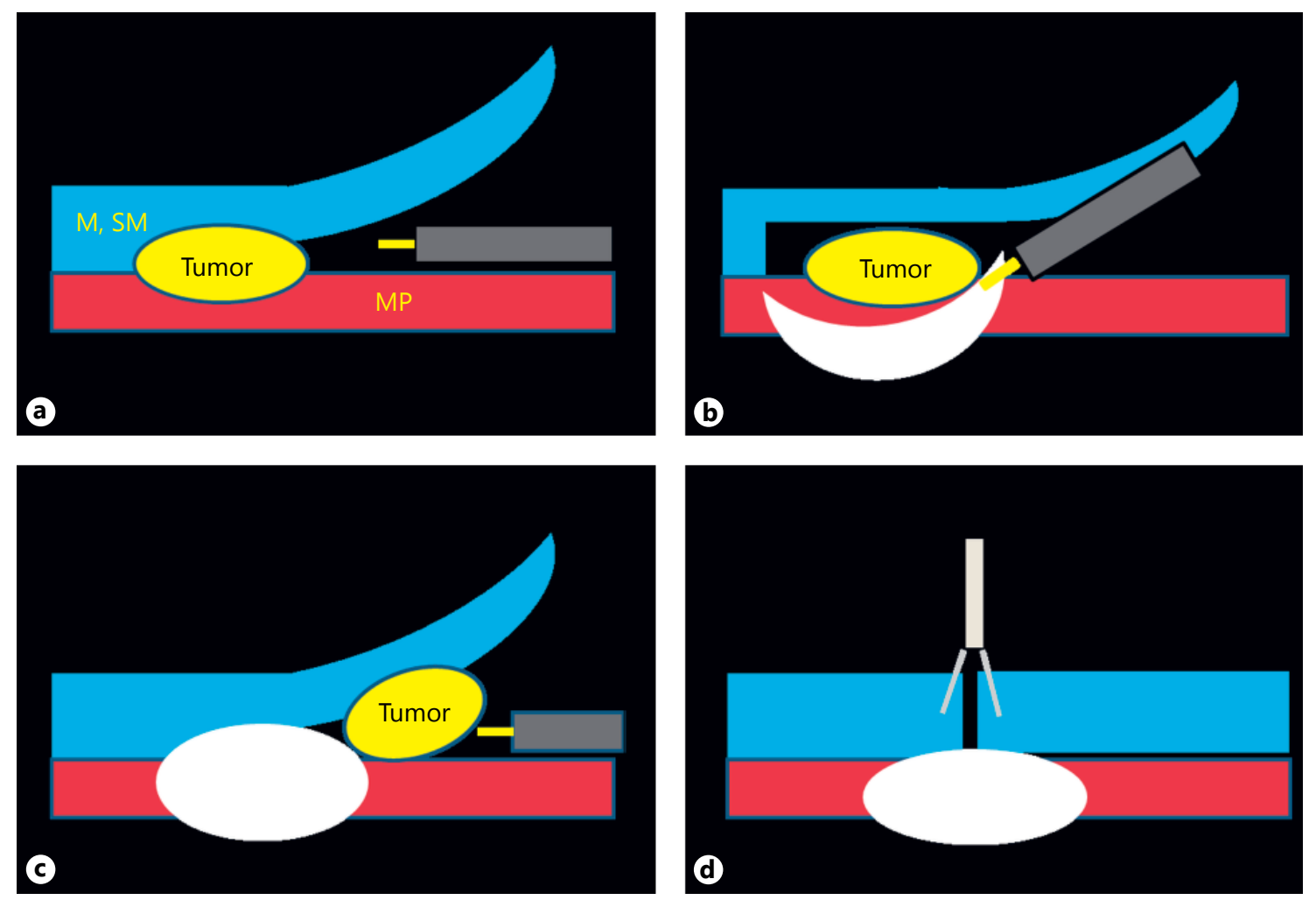

Fig. 2. Scheme of STER. After cutting the mucosal flap, submucosal tunnel was created to reach the SET (a). SET derived from MP was resected leaving mucosa and submucosal layer (b). Resected SET was retrieved through the submucosal tunnel (c). The submucosal tunnel and mucosal flap were closed by hemoclips (d).

spectively. EFTRs were successfully performed (100\%) and no complication was observed such as perforation even 3 months after EFTRs. The tumor size was $12.1 \pm 4.7$ (range 6-20) $\mathrm{mm}$. In the pathological findings, there was 1 case of high-risk GIST (5\%), 18 cases (95\%) were of very low-risk GIST, and 4 cases (17\%) had leiomyomas. Fever (over $37^{\circ} \mathrm{C}$ ), was observed in 3 cases $(13 \%)$ on the operation day and in 1 case (4\%) on the following day of EFTR. Localized peritonitis occurred in 2 patients (9\%). There was no report of postoperative bleeding or perforation [13]. For EFTR performed on lesions at sites measuring up to $20 \mathrm{~mm}$, this method is safe because of the absence of the postoperative risk of a ruptured suture, given that the closure of the resection site is carried out using an OTSC. However, $17 \%$ of cases of fever following an exposed EFTR have been due to the localized peritonitis caused by the intraperitoneal exposure to gastric contents. Bacteriologically, systematic irrigation before EFTR has been reported to be allowed for the prevention of infections [14], suggesting the importance of preoperative systematic irrigation and intraoperative aseptic manipulation.

Feasibility of Neo-EFTR

\section{STER (fig. 2)}

Sumiyama et al. [15] reported that the submucosal tunnel technique was an access route for NOTES. Moreover, this technique was developed as one of the methods of en bloc biopsy [16]. Ye et al. [17] reported a prospective study of STER. The procedure of STER is as follows: first, a $5 \mathrm{~cm}$ submucosal tunnel is created from tumors; second, the tumor is removed using ESD techniques; and third, the mucosal opening is closed with hemoclips. Eightyfive patients with upper gastrointestinal subepithelial tumors (SETs) or SMT were enrolled and they underwent STERs successfully. Among these, in 60 cases, tumors were located in the esophagus; in 25 cases tumors were located on the MP layer in the stomach. The mean tumor size was approximately $20 \mathrm{~mm}$, and the mean operation time was approximately $60 \mathrm{~min}$. Resected tumors were leiomyoma (76\%), GIST (22\%), and others (2\%). Pneumothorax, subcutaneous emphysema, and pneumoperitoneum occurred in 8 patients. The total complication rate was $26.3 \%$ for GISTs and $4.6 \%$ for leiomyomas ( $\mathrm{p}<$ 0.05 ) [17]. From the perspective of infection prevention, this method with a mucosal flap may be more advanced 
than exposed EFTR. However, according to the guidelines published by the Japan Society of Clinical Oncology, a follow-up of the clinical course is recommended for SETs. The following will be the criteria: tumor measuring less than $20 \mathrm{~mm}$, no proliferative tendency suggestive of malignancy, and no change in aspects of the inside of the tumor. In addition, if findings suggest malignancy, priority is given to the determination of the diagnosis using methods such as endoscopic ultrasound-fine needle aspiration. If the diagnosis reveals a GIST, local excision should be performed. In this study, $76 \%$ of the tumors consisted of leiomyomas, which are benign tumors. There were no symptoms such as dysphagia, the average tumor diameter was as small as $20 \mathrm{~mm}$. Moreover, 8 patients (9.4\%) developed pneumothorax, subcutaneous emphysema, and pneumoperitoneum; thus, from the perspective of risk benefit, this result seemed to be controversial [18].

Gong et al. [19] also reported a pilot study of 12 patients (with SMTs located in the esophagus and cardia), which was conducted using endoscopic submucosal tunnel dissection (ESTD), the procedure was more or less similar to STER. The mean size of resected SMTs was around $20 \mathrm{~mm}$, and the mean operation time was around $50 \mathrm{~min}$. However, complications such as pneumothorax and subcutaneous emphysema have been reported in 2 patients (16\% cases) [19-21]. Because of complications arising in ESTD, the procedure is not yet considered safe. In addition, the rate of complications was high in GIST patients and there was a risk of recurrence in the long term. Because, during this procedure, there is some chance to damage the capsule layer or membrane, and there is a possibility of the dissemination of cancer cells as well. Although it has been reported that this method might be considered one of the diagnostic resections, the problem was that benign tumors were larger in number, considering the intensity and enormity of complications [22]. Diagnosis is considered a priority, and subsequently, STER may need to be carried out subsequently.

\section{LECS}

Hiki et al. [23] reported the so called LECS, in which the incision line was determined using a flexible endoscope, and resection opening was closed by hand-sewn suturing using the aparoscopic technique. In Japan, this technique has been approved of and is covered by health insurance [23-30]. Hand and finger disinfection at the time of surgery serves as a reference for infection control. This method is performed to minimize contamination of the surgical site from the common bacterial count of $10^{4}$
$10^{5}$ to $10^{2}-10^{3}$, even when surgical gloves are damaged. Endoscopic irrigation, which could reduce the number of gastric bacteria to $100 / \mathrm{ml}$ or less, would be considered an efficient way of disinfection in the light of surgical disinfection [31-34]. Irrigation with massive amounts of saline (2-3 liters) was used for cleaning in surgery as well as for the debridement of external wounds. This method has been reported to have a prevention effect on bacterial infection, and when irrigation is performed using physiological saline mixed with antibiotics, the mixture has a preventive effect against peritonitis [14].

For SM, massive early gastric cancers with fibrotic ulcer scar, which are difficult to treat by resection using ESD, EFTR with regional lymphadenectomy (such as sentinel lymphadenectomy) has reportedly been carried out using LECS with laparoscopic assistance in order to prevent peritoneal exposure to gastric contents [35]. Exposed EFTR causes a perforation of the gastrointestinal tract, and thus, a collapse of the gastrointestinal tract is inevitable. Therefore, there is a limit to what can be done with pure EFTR, which is performed using only flexible endoscope [36].

As LECS, Neo-EFTR has been reported in various surgical procedures. In those surgical procedures, the lesion is confirmed by using an endoscope, after which a marking of the serosal side of the lesion is carried out using a laparoscope. By suturing the serosa and the muscle layer, the lesion is inverted into the lumen of the gastrointestinal tract. The non-exposed endoscopic wall-inversion surgery (NEWS) is a method that allows for the safe detachment of the lesion endoscopically using a spacer to keep the distance between the lesion and the site of the suture after having sutured the serosa and the muscle layer [37]. The inverted LECS is also a similar procedure [38]. Combination of laparoscopic and endoscopic approaches to neoplasia with non-exposure technique is a surgical procedure in which a laparoscopic resection of the serosa and muscle layer is followed by a pulling of the lesion's mucosa outside the gastrointestinal tract, after which a suture/resection is carried out from the healthy mucosa to the serosa and muscle layer using a linear stapler [39].

The postoperative motility disturbance of the stomach is involved in patients' long-term quality of life (QOL). This is also an important factor in surgical procedures, not only in LECS but also in minimally invasive surgery. The method used for performing full-thickness resection in LECS is different from that used in EFTR. Moreover, the vagal nerve system around the stomach is more injured in LECS than in EFTR. The method of 

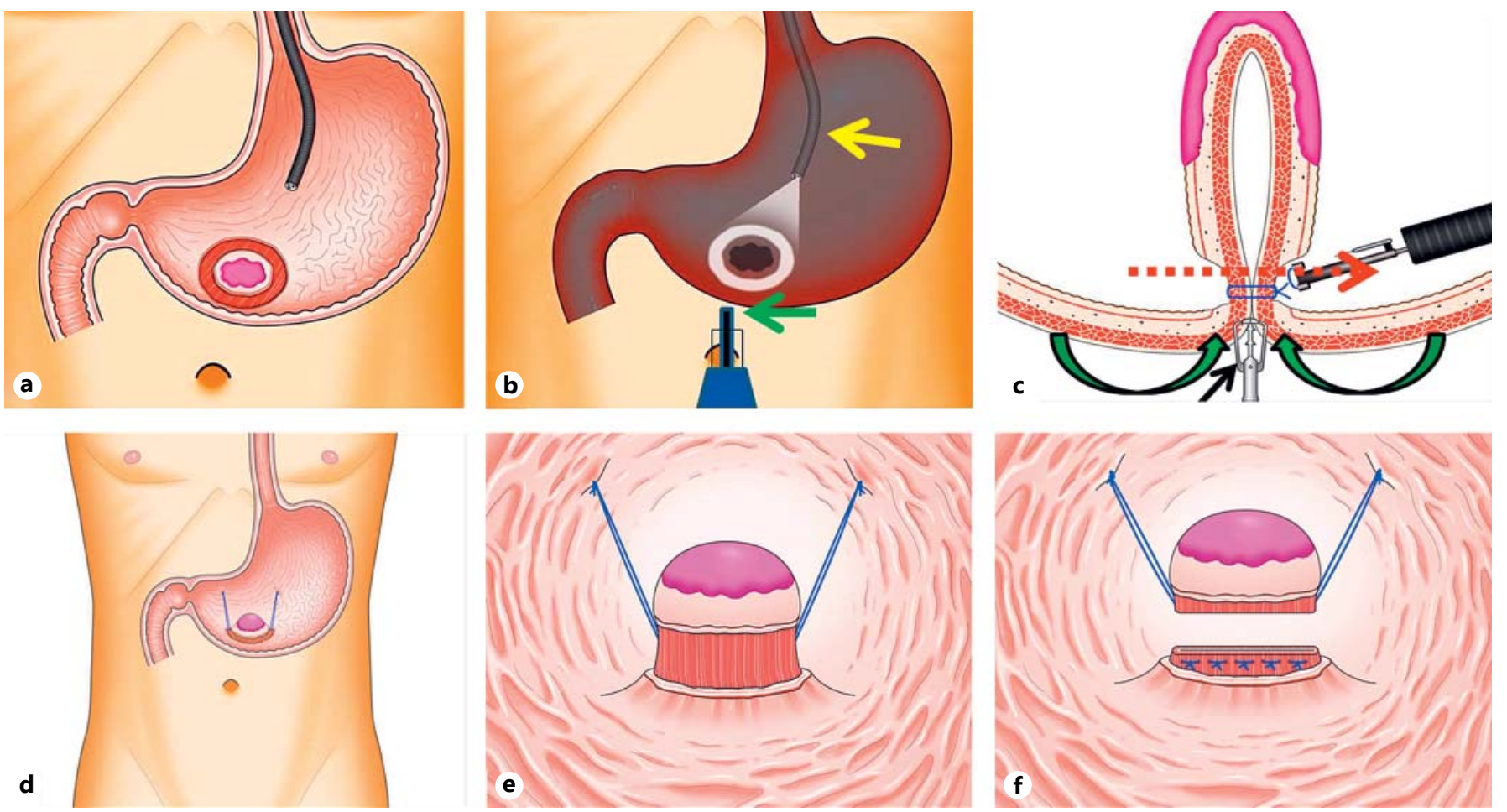

Fig. 3. Scheme of hybrid and pure Neo-EFTR. Hybrid Neo-EFTR: after marking a 30-mm-diameter of pseudo lesion, an 8-mmwidth circular ring reaching the MP was made by ESD method (a). Two endoscopes were used in this method: one through the mouth (yellow arrow), and one via an umbilical blunt port (green arrow). The transmitted light from the endoscope made it possible to detect the lesion through the thin muscular layer (b). After both sides of the edge of the transmitted light were grasped and drawn (green curved arrow) by Twin Grasper ${ }^{\circledR}$ (Ovesco Endoscopy GmbH, Tüebingen, Germany) (black arrow) and sutured by DBSS, fullthickness resection was performed from inside the stomach (red dot arrow, c). Pure Neo-EFTR: a ring-shaped trench $8 \mathrm{~mm}$ in width around the virtual tumor reaching the MP was made with the ESD technique. Using DBSS, a needle penetrated through the exposed muscle layer on the side of the anterior wall. While slowly deflating the air, the needle penetrates through the contralateral gastric mucosa again. The MP on the posterior wall was also lifted up and tied with the gastric mucosa on the opposite side in the same way (d). With increase in insufflation, the stomach expanded; thus, the traction of the thread on the MP increased and the muscle layer at the resection site was inverted (e). After full-thickness suturing of the serosa and the muscle layer was performed with a 3-4-mm pitch, full-thickness resection was performed (f).

ported as one of the reasons for delayed gastric emptying. Therefore, most of the LECS did not have motility disturbance from the point of motility disturbance caused by vagal nerve damage or gastric deformity and had many advantages including minimally invasive local gastrectomy.

\section{Hybrid Neo-EFTR (fig. 3a-c)}

Kim et al. [42] reported the method of resecting a fullthickness gastric wall from inside after laparoscopic seromuscular suturing and inversion of the gastric wall, followed by endoscopic mucosal suturing with endoloops and clips. This surgical procedure has solved the problems of infection and peritoneal dissemination, but it is practically the same as the procedure used in the afore- 
mentioned NEWS [37]. In a previous report of a study using a resected porcine stomach, Mori et al. [7] inserted a flexible endoscope through only one umbilical port. In this study, the serosal side of the tumor was grasped by using a Twin Grasper ${ }^{\circledR}$ (Ovesco Endoscopy $\mathrm{GmbH}$, Tuebingen, Germany), tumor was put in an inverted position, suturing was performed using a fullthickness suturing device designed for flexible endoscopes, and subsequently, resection was conducted. So, this report was a new surgical procedure using 2 flexible endoscopes [7]. However, at sites where the small omentum or the greater omentum was present, the procedure was performed after laparoscopic treatment; thus, this method can also be classified as a type of LECS.

\section{Pure Neo-EFTR (fig. 3d-f)}

Performing a Neo-EFTR using a flexible endoscope throughout the entire process of a surgical procedure is called a 'pure Neo-EFTR'. Although it is essential to have a full-thickness suturing device designed especially for such a method, the procedure is highly feasible because it does not require a counter-traction device. In a NeoEFTR, the air insufflated in the gastrointestinal tract flows, and as a result, it is improbable for the digestive tract to disintegrate or for the operating field to be lost. Therefore, full-thickness suturing devices are more important. In addition, this procedure causes no intraoperative peritoneal exposure of malignant cells of the epithelial tumors and has a higher level of safety with respect to infections. Because of the absence of an efficient fullthickness suturing device for use with flexible endoscopes yet, excising a large lesion using only a flexible endoscope was impossible. Therefore, we have developed the Double-Armed Bar Suturing System (DBSS), a full-thickness suturing device that can be used with flexible endoscopes and we have been using this user-friendly device since 2009. Animal experiments using DBSS have shown that Neo-EFTR might be the procedure with the highest feasibility among other available EFTR techniques. Details about the method of operation of DBSS will be described later.

\section{Development of Suturing Systems for Flexible Endoscope}

The Overstitch System (Apollo Endosurgery, Austin, Tex., USA) is the commercialized device for flexible endoscope, which is a full-thickness suturing device utilizing suture threads for use with flexible endoscopes [43].
Moreover, the OTSC (Ovesco Endoscopy GmbH, Tüebingen, Germany), a clip-shaped full-thickness suturing device, is also commercially available. A number of reports have mentioned about fistulas or closure of iatrogenic perforations and closure of anastomotic leakage after surgery [44-46]. This information suggested that there is no available ideal suturing device. In fact, the overstitch system requires the use of a 2-channel scope, and the OTSC is a clip-type device that cannot be reverted once released. This results in the problem of misfires.

The DBSS was reported by Mori et al. [47]. The arms are available in various sizes depending on the diameter of the resected opening. After suturing using a needle, the first arm is detached, and then, suturing can be performed quickly, simply by replacing the distal portion from the second needle. The basic motions of the DBSS enable the process of suturing to be carried out, only by parallel movements of the first arm, which is put in place with the suture and the second arm to which the puncture needle is attached. The first arm is inserted into the serosal side of the wall of the digestive tract, on the left side of the resection opening. The rails of the first arm, which are placed on the thread on the left side, are coupled with the rails of the second arm (fig. 4a). They penetrate all the layers of the wall of the digestive tract and are joined with the suture on the first arm (fig. 4b). Next, the second arm is rotated toward the right side and is adapted to the rail on the right side of the first arm (fig. 4c). Similarly, the wall of the digestive tract is penetrated in all its layers, and is joined with the suture on the first arm (fig. 4d). Additionally, the first arm also functions as a stopper and prevents injuries on adjacent organs. This allows for putting in place a safe suture. Just by attaching a hood part to the tip of a one-channel, general-purpose endoscope, this structure allows for the forceps channel to be used in various applications (fig. 4e, f).

A number of studies were carried out to examine fullthickness sutures in a resected porcine stomach using a prototype of the DBSS. Leak test (a basic experiment) was carried out on the part of the incision orifice, which was subjected to full-thickness suturing after EFTR in excised porcine stomachs (10 pieces per experiment); and the results showed that for surgical hand-sewn sutures, the median was 3,350 $\mathrm{Pa}(\mathrm{G})$ and the $95 \% \mathrm{CI}$ was $2,470-4,250$ $\mathrm{Pa}(\mathrm{G})$, whereas, for DBSS, the median was 3,665 Pa (G) and the $95 \%$ CI was $1,600-4,400 \mathrm{~Pa}(\mathrm{G})$. No significant difference was found between the surgical hand-sewn sutures and the DBSS $(\mathrm{p}=0.542)$. In animals, a full-thickness excision measuring $40 \mathrm{~mm}$ was performed on virtual lesions in the canine stomach. This was followed by
Mori et al. 

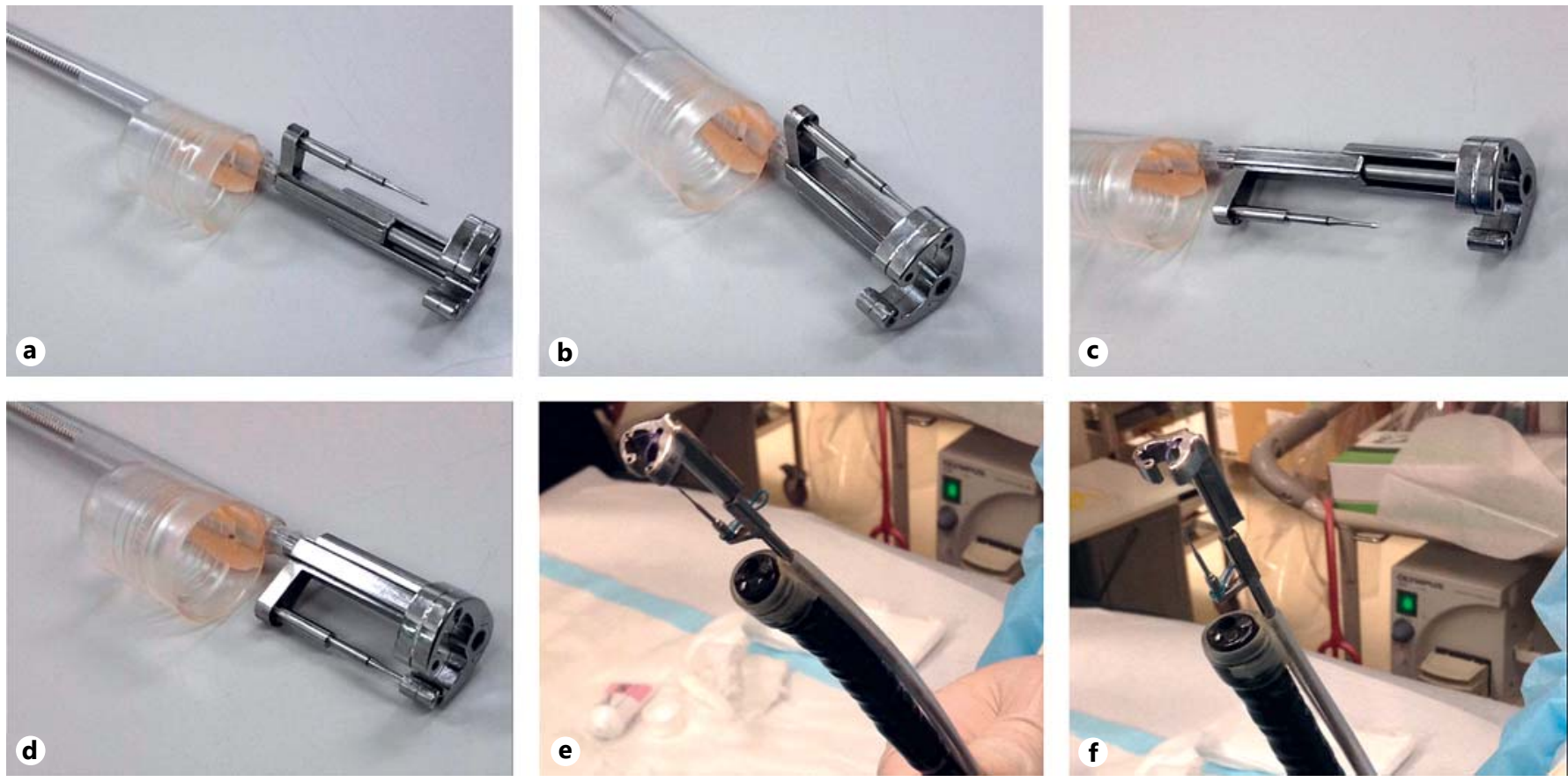

Fig. 4. The rail of the first arm that was connected to the thread on the left side was combined with the rail of the second arm (a). The wall of the digestive tract was penetrated in its full thickness and was bound to the suture thread on the first arm (b). Next, the second arm was rotated toward the right side and was combined with

the placement of 4-mm bite and 4-mm pitch sutures. Hundred percent survival was observed during the first year follow-up, and this vouches for the method's safety.

\section{Future Perspectives}

In a nut shell, the invasiveness of the surgical procedures in the descending order is as follows: open abdominal surgery (laparotomy); laparoscopic surgery; and LECS/EFTR. EFTR, which allows the possibility to access tumors in the gastrointestinal tract through the shortest distance and which causes no tissue destruction, is clearly less invasive than other surgical procedures. In addition, Neo-EFTR might be the most minimally invasive procedure in comparison to exposed EFTR from the perspective of infection, tumor cell dissemination, and compression of other organs due to the insufflated $\mathrm{CO}_{2}$. Conversely, from the physical point of view, the degree of 'invasiveness' after surgery seems similar to be the degree of invasiveness of the surgical procedure itself. However, invasiveness after surgery includes all types of burdens on patients, specifically all physical changes and finan- the rail on the right side of the first arm (c). In a similar manner, the wall of the digestive tract was penetrated in its full thickness and was bound to the suture thread on the first arm (d). DBSS mounted on pan-endoscope. Stored and closed both the 1st and 2nd arms (e). Full open of the 1st arm (f). cial, psychological, and social burdens occurring after surgery. Particularly, EFTR allows for approaching gastrointestinal tumors with a flexible endoscope via the shortest distance through natural orifices. Therefore, EFTR is obviously the most minimally invasive surgical method. The effects of ultra-minimally invasive surgery on reducing the (financial, physical, and psychological) burdens on patients, as well as its reducing effect on medical expenses, are noteworthy. If EFTR can be performed safely using the existing general-purpose flexible endoscopes, it may greatly contribute to the well-being of the afflicted people.

\section{Disclosure Statement}

The authors declare no conflict of interest and no financial arrangement with any company.

\section{Acknowledgments}

The authors acknowledge the role played by Dr. Makoto Oryu in supporting this study. 


\section{References}

1 Yamaguchi N, Isomoto H, Fukuda E, et al: Clinical outcomes of endoscopic submucosal dissection for early gastric cancer by indication criteria. Digestion 2009;80:173-181.

2 Arezzo A, Passera R, Marchese N, et al: Systematic review and meta-analysis of endoscopic submucosal dissection vs endoscopic mucosal resection for colorectal lesions. United European Gastroenterol J 2016;4:1829.

3 Hoteya S, Iizuka T, Kikuchi D, et al: Clinicopathological outcomes of patients with early gastric cancer after non-curative endoscopic submucosal dissection. Digestion 2016;93: 53-58.

4 Dai J, Huang X, Lu B, et al: Evaluation of the expanded criteria of endoscopic submucosal dissection for early gastric cancers: a metaanalysis. Digestion 2015;92:227-235.

$\checkmark 5$ Kalloo AN, Singh VK, Jagannath SB, et al: Flexible transgastric peritoneoscopy: a novel approach to diagnostic and therapeutic interventions in the peritoneal cavity. Gastrointest Endosc 2004;60:114-117.

6 Cai M, Zhou P, Lourenço LC, et al: Endoscopic full-thickness resection (EFTR) for gastrointestinal subepithelial tumors. Gastrointest Endosc Clin N Am 2016;26:283-295.

$\checkmark 7$ Mori H, Kobara H, Nishiyama N, et al: Nonexposure endoscopic full-thickness resection with two flexible endoscopes equipped with a suturing device: ex vivo study. Endoscopy 2015;47(suppl 1 UCTN):E501-E502.

8 Mori H, Kobara H, Masaki T: Novel NOTES techniques and experimental devices for endoscopic full-thickness resection (EFTR). Gastrointest Endosc Clin N Am 2016;26:323334.

-9 Wang H, Feng X, Ye S, et al: A comparison of the efficacy and safety of endoscopic fullthickness resection and laparoscopic-assisted surgery for small gastrointestinal stromal tumors. Surg Endosc 2016;30:3357-3361.

$10 \mathrm{Lu}$ J, Jiao T, Li Y, et al: Facilitating retroflexed endoscopic full-thickness resection through loop-mediated or rope-mediated countertraction (with videos). Gastrointest Endosc 2016;83:223-228.

11 Liu BR, Song JT: Submucosal tunneling endoscopic resection (STER) and other novel applications of submucosal tunneling in humans. Gastrointest Endosc Clin N Am 2016; 26:271-282.

12 Saxena P, Khashab MA: New NOTES clinical training and program development. Gastrointest Endosc Clin N Am 2016;26:385400.

13 Guo J, Liu Z, Sun S, et al: Endoscopic fullthickness resection with defect closure using an over-the-scope clip for gastric subepithelial tumors originating from the muscularis propria. Surg Endosc 2015;29:3356-3362. 14 Mori H, Kobara H, Tsushimi T, et al: Reduc-
tion effect of bacterial counts by preoperative saline lavage of the stomach in performing laparoscopic and endoscopic cooperative surgery. World J Gastroenterol 2014;20:1576315770.

15 Sumiyama K, Gostout CJ, Rajan E, et al: Submucosal endoscopy with mucosal flap safety valve. Gastrointest Endosc 2007;65:688694.

16 Kobara H, Mori H, Rafiq K, et al: Submucosal tunneling techniques: current perspectives. Clin Exp Gastroenterol 2014;7:67-74.

17 Ye LP, Zhang Y, Mao XL, et al: Submucosal tunneling endoscopic resection for small upper gastrointestinal subepithelial tumors originating from the muscularis propria layer. Surg Endosc 2014;28:524-530.

18 Liu H, Wei LL, Zhang YZ, et al: Submucosal tunnelling endoscopic resection (STER) for the treatment of a case of huge esophageal tumor arising in the muscularis propria: a case report and review of literature. Int J Clin Exp Med 2015;8:15846-15851.

19 Gong W, Xiong Y, Zhi F, et al: Preliminary experience of endoscopic submucosal tunnel dissection for upper gastrointestinal submucosal tumors. Endoscopy 2012;44:231-235.

20 Lee SH, Kim SJ, Lee TH, et al: Human applications of submucosal endoscopy under conscious sedation for pure natural orifice transluminal endoscopic surgery. Surg Endosc 2013;27:3016-3020.

21 Inoue H, Ikeda H, Hosoya T, et al: Submucosal endoscopic tumor resection for subepithelial tumors in the esophagus and cardia. Endoscopy 2012;44:225-230.

22 Li B, Liu J, Lu Y, et al: Submucosal tunneling endoscopic resection for tumors of the esophagogastric junction. Minim Invasive Ther $\mathrm{Al}$ lied Technol 2016;25:141-147.

23 Hiki N, Yamamoto Y, Fukunaga T, et al: Laparoscopic and endoscopic cooperative surgery for gastrointestinal stromal tumor dissection. Surg Endosc 2008;22:1729-1735.

24 Namikawa T, Hanazaki K: Laparoscopic endoscopic cooperative surgery as a minimally invasive treatment for gastric submucosal tumor. World J Gastrointest Endosc 2015;7: $1150-1156$

25 Nabeshima K, Tomioku M, Nakamura K, et al: Solitary fibrous tumor of the stomach treated with laparoscopic and endoscopic cooperative surgery. Tokai J Exp Clin Med 2015; 40:120-123.

26 Irino T, Nunobe S, Hiki N, et al: Laparoscopic-endoscopic cooperative surgery for duodenal tumors: a unique procedure that helps ensure the safety of endoscopic submucosal dissection. Endoscopy 2015;47:349-351.

27 Tsushimi T, Mori H, Harada T, et al: Laparoscopic and endoscopic cooperative surgery for duodenal neuroendocrine tumor (NET) G1: report of a case. Int J Surg Case Rep 2014; 5:1021-1024.

28 Waseda Y, Doyama H, Inaki N, et al: Does laparoscopic and endoscopic cooperative surgery for gastric submucosal tumors preserve residual gastric motility? Results of a retrospective single-center study. PLoS One 2014; 9:e101337.

29 Choi SM, Kim MC, Jung GJ, et al: Laparoscopic wedge resection for gastric GIST: longterm follow-up results. Eur J Surg Oncol 2007; 33:444-447.

30 Qiu WQ, Zhuang J, Wang M, et al: Minimally invasive treatment of laparoscopic and endoscopic cooperative surgery for patients with gastric gastrointestinal stromal tumors. J Dig Dis 2013;14:469-473.

31 Swayne SL, Brisson B, Weese JS, et al: Evaluating the effect of intraoperative peritoneal lavage on bacterial culture in dogs with suspected septic peritonitis. Can Vet J 2012;53:971977.

32 Romagnuolo J, Morris J, Palesch S, et al: Natural orifice transluminal endoscopic surgery versus laparoscopic surgery for inadvertent colon injury repair: feasibility, risk of abdominal adhesions, and peritoneal contamination in a porcine survival model. Gastrointest Endosc 2010;71:817-823.

33 Hesami MA, Alipour H, Nikoupour Daylami $\mathrm{H}$, et al: Irrigation of abdomen with imipenem solution decreases surgical site infections in patients with perforated appendicitis: a randomized clinical trial. Iran Red Crescent Med J 2014; 16:e12732

34 Nikfarjam M, Weinberg L, Fink MA, et al: Pressurized pulse irrigation with saline reduces surgical-site infections following major hepatobiliary and pancreatic surgery: randomized controlled trial. World J Surg 2014; 38:447-455.

35 Goto O, Takeuchi H, Kawakubo H, et al: First case of non-exposed endoscopic wall-inversion surgery with sentinel node basin dissection for early gastric cancer. Gastric Cancer 2015;18:434-439.

-36 Mori H, Kobara H, Kazi R, et al: Balloonarmed mechanical counter traction and double-armed bar suturing systems for pure endoscopic full-thickness resection. Gastroenterology 2014;147:278-280.e1

37 Goto O, Mitsui T, Fujishiro M, et al: New method of endoscopic full-thickness resection: a pilot study of non-exposed endoscopic wall-inversion surgery in an ex vivo porcine model. Gastric Cancer 2011;14:183-187.

38 Nunobe S, Hiki N, Gotoda T, et al: Successful application of laparoscopic and endoscopic cooperative surgery (LECS) for a lateralspreading mucosal gastric cancer. Gastric Cancer 2012;15:338-342.

39 Inoue H, Ikeda H, Hosoya T, et al: Endoscopic mucosal resection, endoscopic submucosal dissection, and beyond: full-layer resection for gastric cancer with nonexposure technique (CLEAN-NET). Surg Oncol Clin N Am 2012;21:129-140.

40 Ueda E, Kitayama J, Seto Y, et al: Postoperative complications after local resection of the stomach. Surg Today 2002;32:305-309. 
41 Tomita R, Ikeda T, Fujisaki S, et al: Effects of mosapride citrate on patients after vagal nerve preserving distal gastrectomy reconstructed by interposition of a jejunal J pouch with a jejunal conduit for early gastric cancer. World J Surg 2006;30:205-212.

42 Kim CG, Yoon HM, Lee JY, et al: Nonexposure endolaparoscopic full-thickness resection with simple suturing technique. Endoscopy 2015;47:1171-1174.
43 Kantsevoy SV, Thuluvath PJ: Successful closure of a chronic refractory gastrocutaneous fistula with a new endoscopic suturing device (with video). Gastrointest Endosc 2012;75: 688-690.

44 Schmidt A, Meier B, Caca K: Endoscopic fullthickness resection: current status. World J Gastroenterol 2015;21:9273-9285.

45 Brechmann T, Schmiegel W: Successful treatment of life-threatening bleeding from a duodenal posterior bulb peptic ulcer by an overthe-scope-clip. World J Gastroenterol 2015; 21:1666-1669.
46 Mönkemüller K, Sarker S, Baig KR: Endoscopic creation of an omental patch with an over-the-scope clip system after endoscopic excavation and resection of a large gastrointestinal stromal tumor of the stomach. Endoscopy 2014;46(suppl 1 UCTN):E451-E452.

7 Mori H, Kobara H, Fujihara S, et al: Feasibility of pure EFTR using an innovative new endoscopic suturing device: the double-arm-bar suturing system (with video). Surg Endosc 2014;28:683-690. 